\title{
IV. Das Vorkommen von Schimmel im Magen und dessen wahrscheinliche Bedeutung. ${ }^{3}$ )
}

Von Dr. Max Einhorn, Professor an der N. Y. Postgraduate Medical School and Hospital, New York.

Die Rolle, welche Mikroorganismen (Spalt- nnd Schimmelpilze) beim Zustandekommen von pathologischen Prozessen im Magen spieleu, wird von den Antoren verschieden gedentet. Die meisten Kliniker schreiben ihnen keine besondere Bedenting zil. Audere dagegen schieben sic ziemlich in den Vordergrund, so z. B. Talma (1), der behauptet, dass die durch Mikroorganismen veranlasste Gährung der Kohlehydrate Ursache der Hyperchlorhydric ist. Noch andere legen viel Gewicht, nicht so sehr auf die verschiedenen Arten dieser Mikrobien, als anf ihre eventuellen Mengenverhältnisse. Zn diesen Antoren gehört besonders Naunyn (2).

Die Schimmclpilze als solche finden auf dem Gebiete der Magenaffektione’l wenig Erwähnung. Ich möchte die einzeluen Daten, die ich in der Litteratur vorfand, in Folgendem anführen.

L eube (3) schreibt, wie folgt: „Gewöhnlich findet man Hefe- nnd Schimmelpilze, Sarzine und die verschiedensten sonstigen Spaltpilze. Da diese Pilze aber alle keinen direkt schädlichen Einfluss auf die Magenschleimhaut ausüben und ihre Anwesenheit in kranken Magen die Heilung desselben nicht wesentlich in Frage stellt, so muss das Torkommen der Pilze im Mageninhalt als etwas mehr Zufalliges, als ein Symptom gewisser krankhafter Vorgänge im Magen angesehen werden. In sehr seltenen Fällen dagegen scheinen gewisse Mikroorganismen Entzündung und Geschwürsbildung in Magen anregen zu können; doch sind bis jetzt von anatomischer Seite so wenig Beobacht.ungen iiber diese Gastritis mycotica gemacht, dass von der Aufstellung. eines klinischen Bildes und einer Diagnose derselben vor der Hand besser abgesehen wird."

De Bary (4) hat den Mageninhalt von 17 Magenkranken öfter auf Mikroorganismen untersucht und fand: Fadenpilze: Oidiım lactis (Fall 1, 8), andere Formen, unbestimmte (Fall 1, 3), Mucornycelien (Fall 3, 4), Leptothrix buccalis (Fall 14, 17).

1) Verhandlungen 'des XXVIII. Congresses der deutschen Gesellschaft für Chirnlgie. Referirt Centralblatt für Chirurgie 1899, No. 27.

2) 1. c.

3) Vorgetragen am 1. Mai 1900 in der American Gastro-enterological Association zu Washington, D. C. 
Im allgemeinen constatirt er, dass Soorpilz und Schimmelpilze seltener seien. Niannyı sagt: „Nur beiläıfig erwähne ichı hier das Vorkommen von Schimmelpilzen im Magen. Ich habe in zwei Fällen Schinımelpilze im Magen gefunden: den einen Fall beobachtete ich vor drei Jahren; es laandelte sich nm ein ungefähr 40 jälniges Franenzimnner mit mässigen dyspeptischen Beschwerden und erheblicher Weite und Tiefstand des Magens. Nach zweimaliger Answaschung des Magens entzog sich die Kranke der Behandlung. Die Schimmelpilze, welche das erste Mal reichlich vorhanden gewesen waren, wurden das zweite Mal im Waschwasser nur noch schwierig gefunden.

Der zweite Fall kam mir vor etwa einem Jahre zinr Beobachtming: Ich besitze leider keine Notizen ïbcr denselben und kann nur angeben, dass, wenn ich mich recht entsinne, die Sclimmelpilze nach einmaligem Auswasclien aus dem Mageninhalt verschwanden und dass hier keine erhebliche mechanische Mageninsuffizienz bestand.“

Bei Boas (5) finden sich folgende Bemerkungen, unser Thema betreffend: "Von Sprosspilzen sind (wenigstens in geringen Mengent) als normaler Bestandtheil die Hefepilze zu bezeichnen. Auch Soorpilz (Oidium s. Saccharomyces albicans), nıeist aus der Mundhölle stammend, wird mitunter angetroffen. Desgleichen findet man in klciner Zahl Schimmelpilze (Mucorformen), gleichfalls ohne wesentliche pathognostische Bedeutung."

Pathologisch-anatomisch sind foloende Stellen, die sich bei Kle bs (6) finden, von Belang: „Anders verlielt es sich mit einer Mykose des Magens, die zuerst von E. v. Wahl (Virchow's Archiv Bd. XXI), danı von Recklinghausen (Virchow's Archiv Bd. XXX) beobachtet worden ist, indem hier die Pilze in die Drïsenschlläuche eindrangen. In ersten Falle fanden sich besonders im Pylorustheil zahlreiche Heerde von stecknadelkopforossen pustelartigen Knoten von theils gelber, theils rother F'arbe, welche die Drüsenschicht einnahmen und bis zur Submucosa reichten, im zweiten älnlliche, etwas grössere Heerde mit centraler Nekrose im Fundustheil. In diesem Falle befanden sicl die Pilze sowohl iı den Drüsenschläuchen wie auch stellenweise in dem zwischen denselben befindlichen Gewebc, so dass sie die Enden der Schläuche intereinander verbanden. - In beiden Fällen waren die gleichen Pilzformen gefunden worden: sehr feine, nicht oder nur selten gegliederte Fäden, daneben fcine Körnchen, zuweilen zu rosenkranzartigen Schnïren aneinander gereiht, Formen, die demnach der Leptothrixreihe angehören." - Klebs fïhrt die Nekrose auf eine Wucherung dieser Pilze zurück.

Eichhorst (7) drückt sich in ähnlicher Weise aus: „Oidium albicans kommt im Magen nicht häufig vor. - $\mathrm{R}$ e iss hat cinen derartigen Fall bei einem 18jälırigen Mädchen beschrieben. Es fand sich hier die Magenschleimhaut mit weisslichen, bis stecknadelkopforossen, halbkugeligen Körnern bedeckt, welche stellenweise im Fundus ventriculi zu grösseren Flecken confluirt waren ... Mikroskopisch fand man die Cylinderepithelzellen stellenweise zerstört, die Schleimhatut von Rundzellen reichlich durchsetzt, nirgends ein Eindringen der Pilze in die Blutgefässe. Ausser im Magen traf man noch im Schlunde Soorpilze an. - Rudnew hat bei einer an Cholera verstorbenen Frau Penicillium glaucum gefunden, wobei die Pilze nalıe dem Pylorus zwei kleine Tumoren reichlich durchsetzten. Auch Nauny n beobachtete in zwei Fällen Schimnelpilze im Mageninhalt. Kundrat beschrieb neuerdings bei einem Manne mit Favus universalis Favuskolonieen auf de Magen- und Dickdarmschleimhant, welche zu einer kroupös-diplitherischen Entzündıng und zu Geschwürsbildungen geführt hatten."

Grawitz (8) ist es gelungen, bei Einspritzung von Aspergilluskolonieen in die Blutbalm Pilzheerderkrankungen an der Niere und Leber hervorzurufen. Ueber das Verhalten der Pilze im Magen jedoch findet sichı in dieser Arbeit nichts erwälnt.

In allen den angeführten Litteraturangaben, mit Ansnahme der pathologiscli-anatomischen Daten, beziehen sich die spärlichen Bemerkungen über Schimmelpilze im Magen auf den Befund der mikroskopischen Untersuchung.

Schimmel als solcher, bei makroskopischer Untersuchung mit dem nackten Ange erkennbar, ist meines Wissens klinisch im Magen vorläufig noch nicht beobachtet worden. Jedenfalls findet sich in der Litteratur nichts dariiber erwähnt.

Ich habe nuu Gelegeuhcit gelubt, mehrere Fille von Schimmelbildung im Magen zu sehen, und es scheint der Mïhe werth, auf diesen Gegenstand etwas näher einzugehen. In den Fällen unter meiner Beobachtung fanden sich im Spïlwasser des nüchternen Nagens kleine, bald schwärzlich grane, bald braungrüne Flocken $(2-5 \mathrm{~mm}$ im Durchmesser siehe Fig. 1) in verschiedener Zahl (4-50 und darüber). Die mikroskopische Untersuchung zeigte, dass diese Flocken aus lauter Sporen und Pilzfäden bestanden und beinahe nichts anderes enthielten. Aelıliche Flocken wurden bei denselben Patienten auch im Mageninhalt nach einer Probemahlzeit gefunden, und das Mikroskop zeigte wieder dasselbe Bild wie die Flöckchen des nïchternen Magens.

Manchmal sind diese schwärzlich grauen Massen in Sclleim eingebettet; man sieht dann ausser den Pilzkolonieen Schleimkörperchen und zahlreiche Epithelzellen. Dieses deutet auf das innige Verhältniss zwischen den Funguskolonieen und der Schleimhautoberfläche. Erstere müssen ziemlich fest an diesen haften, vielleicht gar in die Epithel-

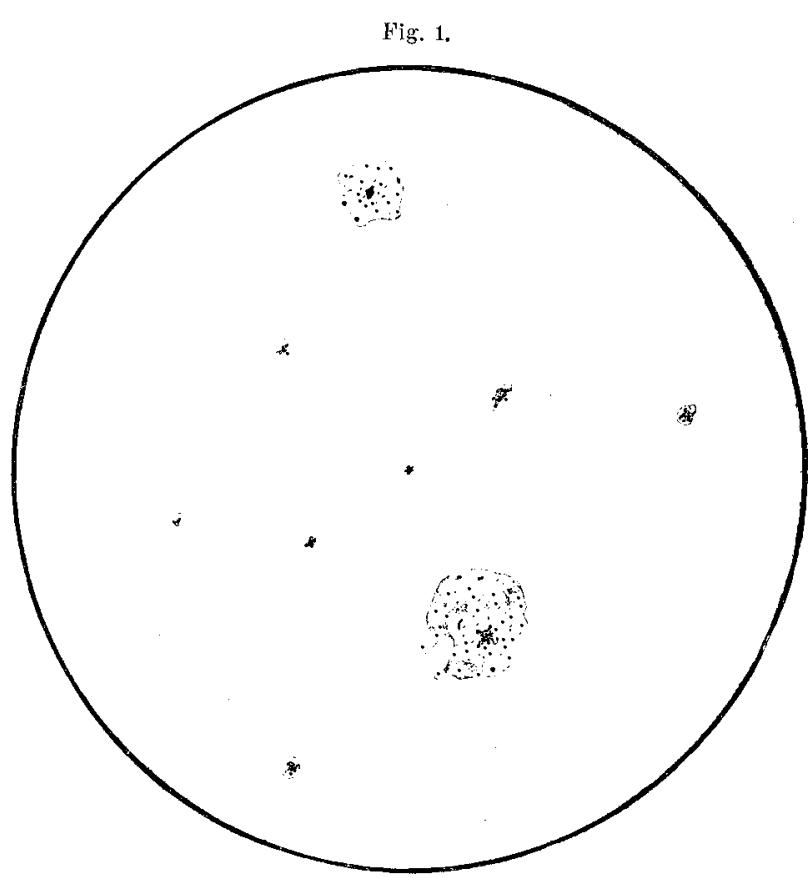

Kleine in Magen vorgefundene Schinmelhäıtchen. Natürliche Grösse.

sclicht hineinwuchern. Dieses feste Anliaften muss auch noch ans folgendem Grunde angenommen werden. Würen die Pilze nur eine zufällige Beimischung der Ingesta. d. h. mit denselben hineingebracht und auch wieder weiter geschafft, ohne dass es zu einer Wucherung derselben käme, dann würde man sie nur im Mageninlıalt nach Mahl zeiten vorfinden, nicht aber im nüchternen Zustande des Patienten, d. l. wo keine Nahrung angetroffen wird. In Wirllichkeit aber kommen diese Flocken in den betreffenden Fällen im nüchternen Magen gerade in grosser Zahl vor.

Sind diese Schimmelpilze, dic im Magen angetroffen werden, noch am Lebell, oder werden sie durch die Aktion des Magensaftes abgetödtet? Diese Frage muss dahin beantwortet werden, dass die Schimmelkolonieen ihr volles Leben haben und der weiteren Entwickelung vollkommen fähig sind. Lässt man nämlich die gefundenen Flöckchen in etwas Wasser oder im Magenfiltrat des betreffeuden Patienten stelıen, dann bemerkt man bald die Bildung von weisslich grauen Wölkchen, welche mit den Flockeu in Zusammenhang stehen und unter dem Mikroskop Sporenkolonieen und Pilzfäden zeigen, von seh ähnlicher Bescìaffenheit wie der ursprüngliche Schimmel

Naclı diesen einleitenden Bemerkungen sei es mir gestattet, einige der beobachteten Fälle genauer zu beschreiben:

Fall 1. Hyperchlorhydrie, nebst periodischer continuirlicher Gastrosuccorrhoea.

October 1899. Wm. R., 38 Jahre alt, litt seit vier Jahren an periodischem Erbrechen von 2-4 Tagen Daner. Wahrend zuerst die Brech-

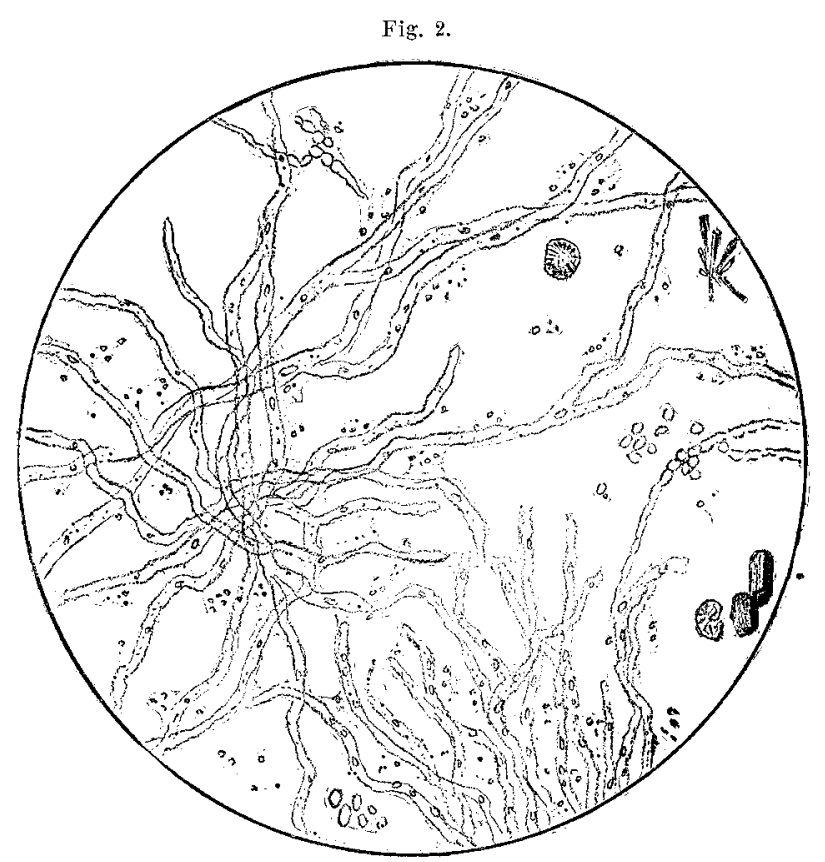

Grünliches Häutchen in Spülwasser des Magens (Wm. R.), im nüchteruen Zustaude gefunden. Man sieht Myzelien, freie Sporen und einige Krystalle, $\times 240$. 
attacken etwa eimmal in vier oder fünf Monaten auftratcn, hatten sic siclı im dritten Jahre der Erkrankung derart celäuft, dass dieselben alle 3-4 Wochen crschicnen. Seitdem fühlte sich Patient auch zwischen den Brechanfällen nicht mehr wohl, indem er stetio etwa zwei Stunden nach den Mablzeiten an intensivem Sodbrennen nud Magenschmerzen laborirte. Verstopfing war gleichfalls in hohen Grade vorhanden. Patient hatte etwa 30 Pfund an Gewicht verloren. Zu dieser Zeit wal er zuerst in meine Behandlung grekommen. Eine genaue Untersuchung ergab, dass es sich um Hyperchlorhydrie mit periodisch anftretcuder continuirlicher Gastrosuccorrhoea liandelte. Patient wurde aul eine passende Diät gesetzt und mit Bromnatrium sowie Alkalien behandelt. Er war dann zehn Monate frei von den Brechanfällen, woranf jedoch dieselben in alter Weise wieder auftraten. Nun consultirte mich $\mathrm{Pa}_{\text {- }}$ tient von nelım, und zwar walhrend einer solchen Attacke.

Status praesens: Patient sieht gut genährt, aber etwas blass aus. An den Brustorganen lässt sich nichts abnormes entdecken. Die Magengegend ist auf Druck etwas empfindlich. Das Kniephänomen ist vorhanden. Urin ist frei von Eiweiss und Zucker.

Im nüchternen Zistande finden sich etwa $60 \mathrm{ccm}$ Magensaft mit etwas Galle vermischt Bei der darauf folgenden Magenallsspiilung worden kleine grïnliche Fetzen in ziemlich grosser Zahl (etwa 20-80) im Waschwasser beobachtet. Ich dachte zunächst, dass es sich IIm kleine mit Gallenfarbstoff imbibirte Schleimflocken handelte, allein die mikroskopisclie Untersuchung zeigte, dass dem nicht so war, sondern dass diese Fetzen aus lauter runden Sporen nnd Pilzfäden bestauden. (Fig. 2 und 3.)

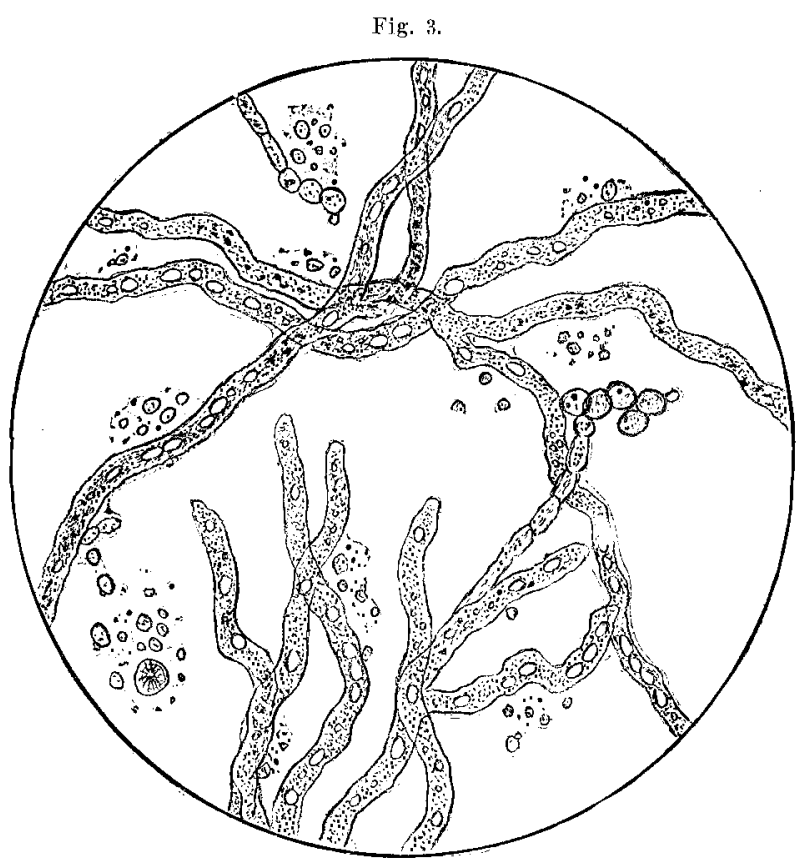

Dasselbe wie Fig. 2, stark vergrössert. $\times 420$

Fall 2. Hyperchlorhydrie.

18. Februar 1900. T. M., 22 Jahre alt, litt seit sechs Jahren an Verdanungsstörıngen; er pflegte viel aufzustossen; die Zunge war immer belegt, und Patient hatte des Morgens häıfjg einen schlechten Geschmack im Munde. Eine halbe bis zwei Stunden nach den Mahlzeiten trat häıfig ein Gefühl von Brennen in der Iler"zgrube anf, und Patient hatte die Empfindung, als ob er einen Fremdkörpcr verschluckt hätte. Im Halse hatte er häufig ein rauhes Gefiihl und musste sich öfter räuspern. Stıllgang war stets regelmässig, Appetit gut, kein Erbrechen, kein Gewichtsverlust.

Status praesens: Patient ist kraftig gebaut und wohl genährt. Die Zunge ist mit einem grünlich-granen Belag ïberzogen. Die Brustorgane zeigen normale Verhältnisse. Am Abdomen lässt sich gleichfalls nichts abnormes entdecken. Knicphänomen ist intakt. Der Urin ist frei von Zucker und Eiweiss.

Die Untersuchung des Magens eine Stunde nach dem Probefrïhstiick ergiebt: $\mathrm{HCl}+$ Azidität $=76$; freie $\mathrm{HCl}=56$

Im Speisebrei sind kleine grïnliche Flocken, Petersilie vergleichbar, in geringer Zahl (etwa vier solche Stückchen auf dem Filter) vorhanden. Diesellen machen zunächst makroskopisch den Eindruck, als $\mathrm{ob}$ es sich entweder um geringe Partikelchen grtinen Gemüses oder um mit Gallenfarbstoff imbibirte Schleimflocken handle. Das Mikroskop zeigt jedoch, dass diese Flocken Sclimmelpilzkolonieen darstellen, aus Sporen und Fäden bestehend. (Fig. 4.)

Patient wird am folgenden Morgen im nüchternen Zustande nntersucht. Der Magen enthält keine Speisen, wohl aber eine geringe Menge (20 ccm) Magensaft. Die vorgenommene Ausspïlung reigt im Waschwasser eine erhebliche Anzahl (50-50) der eben bescliriebenen grünlichen Flocken; ausserdem eine geringe Menge Schleim.

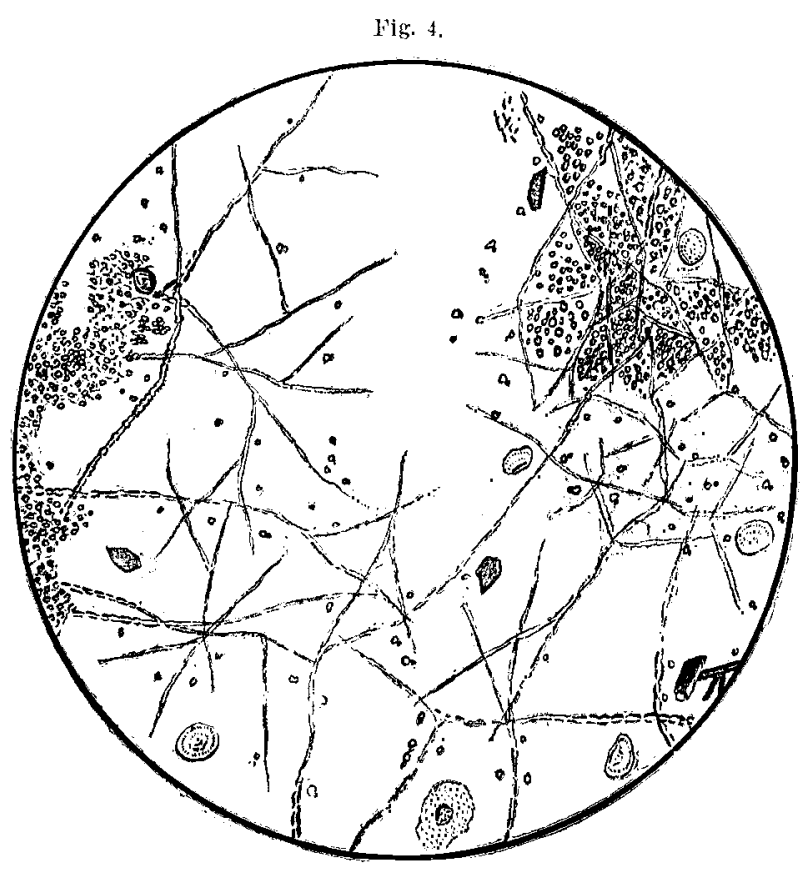

Ein schwär"zliches Flöckchen im Mageninhalt (T. M.), nach Probefrïhstiick vorgefumden. Man sieht zahlreiche Sporen, Myzelien, einige Krystalle, Stärkekörnchen und Epithelzellen. $\times 140$.

Patient wird mit doppeltkohlensaurem Natron und mit Magenansspïlungen, nebst Argentumnitricum-Spray behandelt. Sein Zustand bessert sich, d. h. die subjektiven Symptome lassen nach, nud im Spiilwasser findet man, etwa 14 Tage nach begonnener Behandlıng, entweder gar keine griinlichen Flocken, oder nur selır wenige; einc kleine Menge gelbbrannen Schleimes ist jedoch fast immer im Spülwasser vorhanden, und in demselben lassen sich Epithelzellen und ausserdem Sporen (wohl von Pilzen) nachweisen.

Fall 3. Hyperchlorhydrie.

15. März 1900. George D., 48 Jahre alt, war stets cresund bis etwa vol einem Jahre, wo er an Verdauungsbeschwerden zu leiden begann. Zinächst stellten sich Schmerzen einige Zeit nach dem Essen ein, dann gesellte sich ab und zu anftretendes Erbrechen dazı. Appetit war an fangs ungestört, späterhin schleclit; Stuhlgang angehalten; der Geschmack im Munde häufig sauer. - Patient verlor während seines Leidens 45 Pfund an Gewicht und fühlte sich schwach und elend.

Status praesens: Patient, von kräftigem Bau, sieht blass und elend aus, seine Zunge ist stark belegt. Brustorgane sind intakt. Die Untersuchung des Abdomens ergiebt eine leichte Fmpfindliclikeit der Magengegend ant Druck, Vorhandensein von Plätschern, welches bis etwa zum Nabel erzeugt werden kann, und Fehlen irgend eines Tumors. Die Kniereflexe sind vorhanden. Der Urin enthält weder Zucker noch Eiweiss.

Die Untersuchung des Magens eine Stunde nach dem Probefrühstiick ergiebt: $\mathrm{HCl}+$ Azidität $=104$; freie $\mathrm{HCl}=80$

Nüchtern findet sich der Magen leer; im Spülwasser sind zahlreiche Schimmelflocken (mikroskopisch bestätigt) vorhanden.

Patient wurde mehrere Tage mit Magenausspülıngen und Alkalien behandelt, ansserdem wurde er anf häufige Mahlzeiten leichter Kost gesetzt. Sein Zustand besserte sich; die Schmer'zen wurden geringer das Erbrechen sistirte, und er begann an Gewicht wieder zulmnelumen. Nach einer dreiwöchentlichen Behandlung konnten keine Schimmelhäıtchen mehr im Magen entdeckt werden.

Fall 4. Gastritis gland. chronica und Magenerosionen.

12. Januar 1900. Leopold C., 27 Jahre alt, leidet seit zwei Jahren an dyspeptisclien Symptomen: Druckgefïhl und leichten Sclimerzen in der Regio gastrica etwa eine halbe Stunde nach den Mahlzeiten, häufigem Aufstossen und Verstopfung geringen Grades. Patient hat eine strenge Diät innegehalten und dabei 30 Pfund an Gewicht verloren, während die Beschwerden unverändert fortbestelien.

Status praesens: Patient sieht blass aus; Lippen- und Wangenschleimhaut sind anämisch; Zunge nur wenig belegt. Brustorgane zeigen normale Verhältnisse. Die Palpation des Abdomens ergiebt eine geringe Schmerzhaftigkeit der Epigastralgegend auf Druck.

Die Untersuchung des Magens eine Stunde nach dem Probefrühstïck zeigt: $\mathrm{HCl}+$ Azidität $=24$; eine ziemliche Menge Schleim und zwei schwälzlich-grüne Flocken, die sich bei mikroskopischer Untersuchung als aus Sporen und Schimmelpilzen bestehend erweisen

Im nüchternen Zustande wird der Magen leer befunden: das Waschwasser enthält jedoch eine reichliche Anzahl dieser Schimmelflocken (20-30) und ausserdem vier kleine Schleimhantstückchen (Fig. 5).

Derselbe Befund wurde öfter constatirt, mit dem Unterschiede, dass nach längerer Behandlıng, die in Magenspülung und Anssprayung 


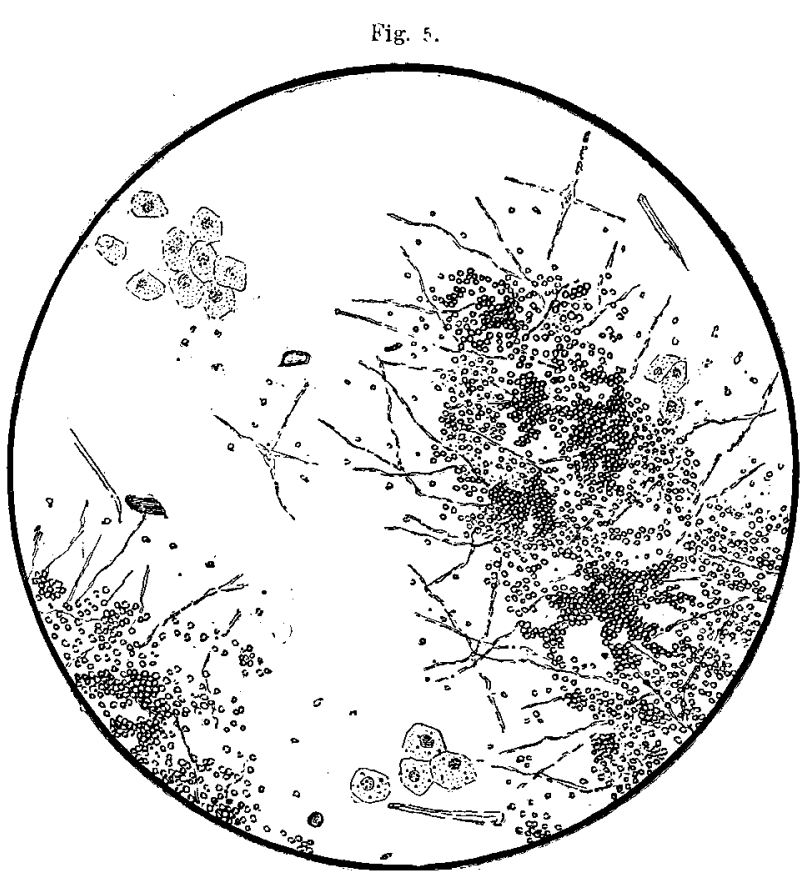

Ein schwärzliches Häutchen, im Spülwasser des nüchternen Magens (Leopold C.) angetroffen. Man sieht zahlreiche Sporenkolonieen, Myzelien, einzelne Krystalle, Epithelzellen und einige Algen. $\times 120$.

mit Höllensteinlösung bestand, die Schlcimhautstiickchen und auch die Schimmelflocken in geringeren Zahlen auftraten.

Patient fülltte sich besser, war kräftiger und hatte weniger $\mathrm{Be}$ schwerden. Ganz frei von seinen Schmerzen ist er jedoch noch nicht.

Ausser den eben genan skizzirten Beobachtungen habe ich noch zwei Fälle von Schimmel im Magen gesehen; in dem einen handelte es sich um eine ausgesprochene langiährige Hyperchlorhydrie; in dem anderen um eine Atonie des Magens mit ziemlich normalem Chemismus und Gastralgieen. Beide Patienten waren nicht lange genug unter Beobachtung, so dass ich über den weiteren Verlauf nicht genügend unterrichtet bin

Dass die vorgefundenen graugrünlichen oder grauschwärzlichen Flocken Schimmelhäutchen darstellen, zeigt die mikroskopische Untersuchung ohne weiteren $Z_{w}$ eifel. Man sieht stets eine ungehener grosse Anzahl von Sporen und Myzelfäden. In allen meinen Fällen war das mikroskopische Bild dasselbe, und liess sich daher annehmen, dass die gefundenen Schimmelpilze ein und derselben Gattung angehören. Um letztere zu bestimmen, habe ich einige dieser grünlichen Häutchen Herrn Prof. Dr. E. K. Dunham zur genaueren Prüfung übergeben, und macht es mir ein grosses Vergniigen, seinen Bericht hier anzuschliessen

Carnegie Laboratorium.

New-York, den 27. April 1900.

Werther Dr. Einhorn! Dic Probe Mageninhalts, welche Sie mir behufs Untersuchung zusandtell, enthielt kleine Partikelchen von schmutzig gelber oder grünlicher Farbe. Unter dem Mikroskop zeioten diese Partikelchen eine granulirte Substanz von unbestimmtem Charakter, ans der Myzelfäden in die umgebende Flüssigkeit austraten. In der letzteren befanden sich zahlreiche runde Körperchen, welche als Sporen von einer Schimmelart aufgefasst wurden. Zeichen von Truktifikation wurden jedoch an den Myzelien vermisst. Versuche, die $\mathrm{My}$ zelien auf Agaragar, Gelatine. Milchzuckerbouillon und auf sauren Medien zu kultiviren, ergaben keinen fruchttragenden Schimmel. Das ursprüngliche Material wurde endlich auf sterilisirtes Brod vertheilt. Auf diesem Medium entwickelten sich Kolonieen von einem Aspergillus, einem Penicillinm und eine Mncorkolonie ohne weitere Schwierigkeit.

Das Penicilljum war scheinbar das gewöhnliche Penicillium glancum. Die Aspergillenkolonieen waren gleichfalls von grüner Farbe. Lediglich geschlechtslose Sporenbildung kam zur Beobachtung.

Ich lege wenig Gewicht anf diese Beobachtungen, da die Brodkulturen erst eine Woche nach Empfang des Materials gemacht worden sind. Es ist somit die Möglichkeit gegeben, dass die gewachsenen Knlturen Verunreinigungen des auf das Brod verstrichenen Materials ihren Ursprung verdanken.

Ich lege drei Zeichnungen bei, welche von dem ursprïnglichen Material mit Hilfe einer Camera lucida gemacht worden sind. (Siehe Fig. $6,7,8$.) Ihr ergebener Edward K. Dunham.

An dieser Stelle sei es mir gestattet, Herrn Prof. Dr. E. K. D unham und Herrn Dr. Goldhorn für die gütige Tntersuchung der Schimmelflöckchen bestens zu danken.

Kehren wir nun zu unseren Fällen, in denen der Schimmel häufig im Magen vorgefunden wnrde, zurück, so drängt sich uns die Frage auf: welche Bedeutung diesen Schimmelpilzen in der Pathologie des Magens zukommt?

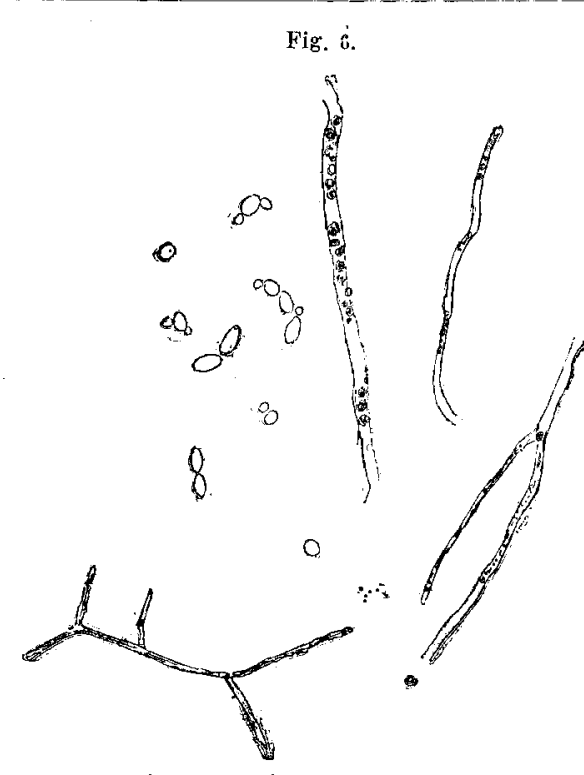

Oc. 2. Obj. $1 / 12$ Oelimmersion. Leitz.
Wenu auch einzelne Pilze im Magen ohne Schaden eine kurze Weile existiren mögen, so finden dieselben normal daselbst keinen günstigen Boden zur Weiterentwickelung; sie werden mit dem Speisebrei innig vermischt und lebendig oder todt durch den Pylorus weiter geschafft. Ganze Pilzkolonieen, schon makroskopisch bemerkbar. wird man daher wohl fast nie in einem normalen Magen antreffen. Ein erhebliches Wachsen ron Schimmel dürf te nur dann möglich sein, wenn eine Pilzkolonie sich in eine Falte der Schleimhautoberfläche des Magens eingenistet hat und derart fest klebt, dass die Weiterbewegung des Chymus dieselbe nicht mitzinschleppen vermag. In diesem Falle komm es zu einem ungestörten Gedeihen der Pilzansiedelung, und beträchtliche Strecken der Magenschleimhaut können nur mit Schimmel bedeckt sein. In unseren oben geschilderten Fällen muss es sich um ein derartiges Vorkommniss handeln. Bei der Ausspülung des Magen übt das hineinströmende Wasser eine ziemliche Kraft ans und reisst manche Schimmelinseln von ihrer Grundlage los, so dass sie jetzt im Waschwasser erscheinen.

Es ist wohl kaum denkbar, dass ein derartiger Schimmelüberzug einzelner Zonen der Magenmucosa ohne Störıng anf die Funktionen des Magens sein sollte. Reizzustände und auch entzündliche Vorgänge dürten sich a priori schon von der mechanischen Wirkung des Schimmels erwarten lassen.

Nach diesen theoretischen Ueberlegıngen dürfte es zweckmässig sein, die beschriebenen Fälle genauer zu priifen und zı.erwägen, ob die Schimmelbildung in ursächlichem Zusammenhang mit den Krankheitserscheinungen stehe. Die Entscheidung dieser Frage stösst jedoch anf grosse Schwierigkeiten, weil das post hoc doch nicht immer auf ein propter hoc schliessen lässt. - Wir haben die Schimmelbildung vornehm-

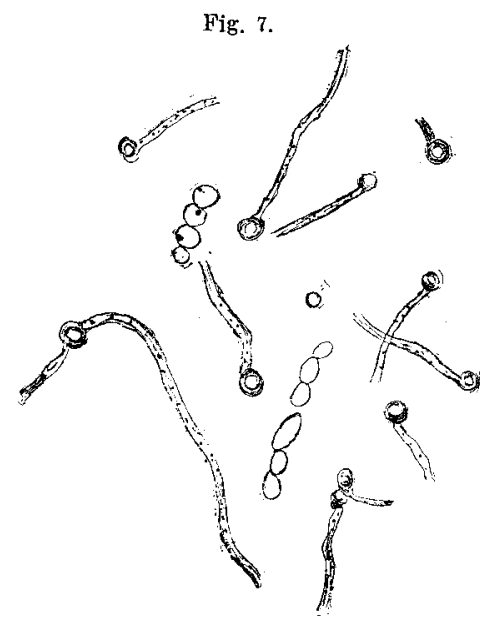

Oc. 2. Obj. $1 / 12$ Oelimmersion.

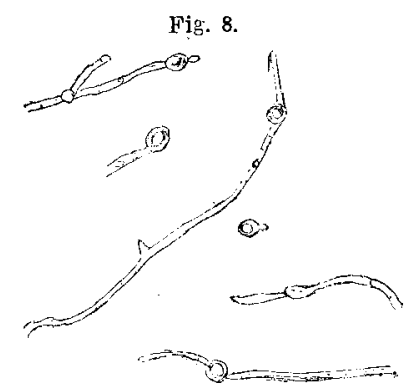

Oc. 2. Obj. ${ }^{1 / 12}$ Oelimmersion.

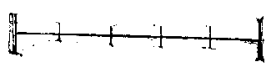

Scala $0,05 \mathrm{~mm}$.

jede Abtheilung $=0,01 \mathrm{~mm}$ lich in zwei Gruppen von Magenerkrankungen angetroffeu, erstens bei intensiver Hyperchlorhydrie (7.11weilen auch mit Hypersekretion und Erbrechen verbunden), und zweitens bei Gastralgieen mit normaler oder herabresetzter Magensaftsekretion. Es lässt sich nicht leugnen, dass in vielen dieser Fälle nach Magenspülungen mit daranf folgender Besprayung mit $1-2 \%$ Lösung von Argentım nitricum die Schimmel flocken geringer wurden, resp. verschwanden und dass dabei anch eine subjektive Besserung im Zustande des Patienten zu constatiren war. Und doch lässt sich nicht mit Sicherheit sagen, dass der Schimmel die bestehenden pathologischen Verhältnisse des Magens geschaffen hat Finden wir doch ganz analoge Fälle ohne die Anwesenheit der Schimmelpilze. Trotzdem erscheint es plausibel, dass diese Schimmelpilze sich in einem gewissen Connex mit den angeführten abnormen $\mathrm{Zu}$ ständen befinden und - falls sie nicht deren Ursache sind - doch dieselben sicherlich steigern.

Das Vorkommen der Schimmelpilze im Magen in grossen Massen dürfte demnach therapeutisch von Belang sein. Man wird danach trachten müssen, den Magen von denselben möglichst zu befreien. Dies geschieht am besten durch Magenspülungen im niichternen Zustande 
des Patienten; dieselben wirken rein mechanisch, indem Schinmelpartieen mit dem Wasser fortgerissen werdell. Auch Berieselungen des Magens mit der Magendouclie dürften in derselben Hinsicht güustig wirken. Nachfolgendes Besprayen des Organs mit einer antiseptischen Lösung (wie Silbernitrat) erscheint gleichlalls von einigem Belang zu sein. Ausser den soeben gezeichneten therapeutischen Maassnahmen wird sich die Behandlung dieser Fälle nach der speziell vorliegenden Erkrankung richten.

$$
\text { Litteratur. }
$$

1. Talma, Von der Gährung der Kohlehydrate im Magen. Zeitschrift für klinische Medicin 1898, Bd. XXXV, S. 542. - 2. B. Nan ny 1 , Ueber das Verhältniss der Magengährungen. Deutsches Archiv für klinische Medicin Bd. XXXI. - 3. W. v. Leube Spezielle Diarnose innerer Krankheitell. Leipzig, 1895. Bd. I, S. 256. - 4. de Bary, Beitrag zur Kenntuiss der niederen Organismen in Mageninlalt. A rchiv für experimentelle Pathologie und Therapie Bd. 20, S. 243. - 5. I. Boas, Magenkrankheiten Theil I, S. 218. - 6. E. Klebs, Handbuch der pathologischen Anatomie Bd. I, S. 201. Berlin 1869. - 7. Eichhorst, Handbuch der speziellen Pathologie und Therapie 1890. Bd. II. S. 170 und 171. - 8. P. Grawitz, Ueber Schiminelvegetationen im melschlichen Organismus. Tirchow's Archiv Bd. LXXXI, S. 355. 